\title{
Producción de biomasa y calidad nutricional de tres forrajes cosechados a dos alturas ${ }^{1}$
}

\author{
Biomass yield and nutritional quality of three forages harvested at two \\ cutting height
}

\author{
Jorge Alberto Elizondo-Salazar ${ }^{2}$
}

\begin{abstract}
Resumen
El objetivo de este trabajo fue determinar el efecto que tiene la altura de cosecha sobre el rendimiento y calidad nutricional en tres especies forrajeras utilizadas para alimentación de ganado lechero. El estudio se llevó a cabo en la Estación Experimental "Alfredo Volio Mata" de la Universidad de Costa Rica. Las especies evaluadas fueron maíz criollo (Zea mays), sorgo negro forrajero (Sorghum almum) y pasto king grass cultivar Taiwán (Pennisetum purpureum), cosechadas a dos alturas de corte (15 y $45 \mathrm{~cm}$ sobre el nivel del suelo). El rendimiento del forraje verde fue diferente $(\mathrm{P}<0,05)$ dentro de cada especie y el de materia seca (MS) disminuyó del 17 al 42\%, al aumentar la altura de corte. Cuando la cosecha se realizó a $15 \mathrm{~cm}$, el maíz, el sorgo negro forrajero y el pasto Taiwán presentaron un rendimiento de materia verde de 51,3, 31,4 y 70,9 t/ha/corte, respectivamente. Con respecto al rendimiento de materia seca (MS), el pasto Taiwán presentó la mayor producción con 11,6 t/ha/corte, cuando se cosechó a 15 cm, mientras que el maíz presentó un rendimiento de 5,4 t/ha/corte cuando se cosechó a $45 \mathrm{~cm}$. El sorgo negro forrajero mejoró significativamente $(\mathrm{P}<0,05)$ su perfil nutricional al incrementar la altura de cosecha. La concentración de MS se incrementó de 22,79 a 25,53\%, la proteína cruda pasó de 8,24 a 12,90\%, mientras que la fibra neutro detergente disminuyó de 63,69 a 59,47\% y la fibra ácido detergente pasó de 43,15 a 37,30\%.
\end{abstract}

Palabras clave: rendimiento forrajero, alimentación animal, pastos tropicales.

\begin{abstract}
The objective of this experiment was to determine the effect of cutting height on yield and nutritional quality of three forage species used for feeding dairy cattle. The study was carried out at the "Alfredo Volio Mata" Experiment Station of the University of Costa Rica. The evaluated species were: native corn (Zea mays), black sorghum (Sorghum almum) and king grass cultivar Taiwan (Pennisetum purpureum); these three species were harvested at two different cutting heights (15 and $45 \mathrm{~cm}$ above ground). Green forage yield was significantly different $(\mathrm{P}<0.05)$ within each of the species and dry matter (DM) yield was reduced between 17 to $42 \%$ when cutting height was increased. When cutting height was done at $15 \mathrm{~cm}$ above ground, corn, black sorghum and Taiwan yielded 51.3, 31.4 and 70.9 t/ha/ cycle, respectively. With regard to DM, the forage species that yielded the most when harvested at $15 \mathrm{~cm}$ above ground was Taiwan grass, with $11.6 \mathrm{t} / \mathrm{ha} /$ cycle, while the forage species that yielded the least when harvested at $45 \mathrm{~cm}$ was corn with only 5.4 t/ha. Black sorghum significantly $(\mathrm{P}<0.05)$ improved its nutritive value when cutting height was
\end{abstract}

1 Recibido: 16 de marzo, 2016. Aceptado: 18 de agosto, 2016. Este trabajo formó parte del proyecto de investigación No 737-B5-188, inscrito en la Vicerrectoría de Investigación de la Universidad de Costa Rica (UCR), San José, Costa Rica.

2 Universidad de Costa Rica, Facultad de Ciencias Agroalimentarias, Estación Experimental Alfredo Volio Mata. Cartago, Costa Rica. jorge. elizondosalazar@ucr.ac.cr 
increased. DM concentration increased from 22.79 to $25.53 \%$, crude protein also increased from 8.24 to $12.90 \%$, meanwhile neutral detergent fiber decreased from 63.69 to $59.47 \%$ and acid detergent fiber also decreased from 43.15 to $37.30 \%$.

Keywords: forage yield, animal nutrition, tropical forages.

\section{Introducción}

Los sistemas productivos, tanto de leche como de carne, se basan en la utilización de forrajes como principal fuente de alimentación, y pese a que, en comparación con el costo de los alimentos balanceados, es un recurso alimenticio más económico, los productores deben hacer un uso eficiente de los mismos y esto tiene que ver con intensificar la producción por área y mejorar la calidad nutricional de los mismos.

Los forrajes tropicales se caracterizan por poseer un valor nutricional medio a bajo, especialmente debido a su baja concentración de proteína cruda y alta porción de componentes de la pared celular, que limitan la producción de proteína microbial en el rumen (Villareal et al., 2006).

Tanto el rendimiento como la calidad nutricional de los forrajes, se ven afectados por una serie de factores internos y externos. Dentro de los internos se encuentra la especie o cultivar utilizado y la edad fisiológica, entre otros. Con respecto a los externos, se puede mencionar el clima, las características físico-químicas del suelo, la edad de corte, la fertilización y otros factores de manejo (Bernal, 1991; Buxton y Fales, 1994; Sheaffer et al., 1998).

Dentro de los factores de manejo, la altura sobre la superficie del suelo a la que se cosecha la planta, afecta el rendimiento y también podría ejercer un efecto sobre la calidad (Neylon y Kung, 2003). Esto adquiere sentido cuando se toma en consideración que en los últimos años se ha desarrollado un interés particular en cosechar las plantas a una altura mayor de lo acostumbrado, con la finalidad de que se mejore la calidad del forraje para ofrecer a los animales (Wu y Roth, 2005; Kung et al., 2008).

Se ha observado, por ejemplo, que cortar las plantas a una altura superior a los $15 \mathrm{~cm}$, puede incrementar la calidad del material a ensilar, principalmente en un esfuerzo para mejorar la digestibilidad de la fibra detergente neutro y la concentración de energía (Neylon y Kung, 2003).

La mayoría de trabajos que se han realizado para estudiar el efecto de la altura de corte sobre el rendimiento y composición nutricional, se han llevado a cabo con plantas de maíz, ya que esta especie se adapta a diferentes condiciones ambientales, brinda una alta concentración de nutrientes, se ensila de forma adecuada y se incorpora fácilmente a las raciones totales mezcladas. Aprovechando estas características del maíz y los estudios que se han realizado con él, se analizaron los datos de once experimentos en los que la planta de maíz para ensilaje fue cosechada a una altura baja o alta (Wu y Roth, 2005); los autores reportaron que cortar a una altura cercana a los 50 cm del suelo incrementó el contenido de proteína cruda, energía neta de lactancia, la digestión de la fibra detergente neutro y la cantidad de leche producida por tonelada de ensilaje ofrecido, cuando se comparó con forraje cosechado a una altura de corte de $15 \mathrm{~cm}$ sobre el nivel del suelo.

En Costa Rica, diversos estudios han evaluado el rendimiento y calidad de diferentes cultivares de maíz para ser utilizados en la alimentación animal (Elizondo y Boschini, 2001; Elizondo y Boschini, 2002; Elizondo y Boschini, 2003; Boschini y Elizondo, 2004a), y solamente un ensayo evaluó el efecto de aumentar la altura de corte sobre la producción y calidad nutricional (Elizondo, 2011). Sin embargo, dicho estudio evaluó el efecto de la altura de corte en solamente una especie forrajera.

El objetivo de este trabajo fue determinar el efecto que tiene la altura de cosecha sobre el rendimiento y calidad nutricional en tres especies forrajeras utilizadas para alimentación del ganado lechero. 


\section{Materiales y métodos}

El experimento se llevó a cabo en la Estación Experimental Alfredo Volio Mata de la Universidad de Costa Rica, ubicada en el Alto de Ochomogo en la provincia de Cartago, con coordenadas geográficas 955' 10' latitud norte y 8357'20" longitud oeste. Su altitud es de $1542 \mathrm{msnm}$, con una precipitación media anual de $2050 \mathrm{~mm}$, distribuida de mayo a noviembre, y una humedad relativa media de $84 \%$. La temperatura media anual es de 19,3 ${ }^{\circ} \mathrm{C}$, con una máxima de $23{ }^{\circ} \mathrm{C}$ y mínima de $13{ }^{\circ} \mathrm{C}$. El suelo se clasifica como Tipic Dystrandepts (Vásquez, 1982), formado por cenizas volcánicas recientes, con una textura franco arcillo arenoso, un pH de 5,8 y un contenido de materia orgánica de 6,24\%; con vocación agrícola limitada por la topografía.

Para las labores de siembra, se araron y rastrearon tres hectáreas de terreno, en las que se sembró maíz criollo amarillo forrajero (Zea mays) adaptado a la zona de Cartago, sorgo negro forrajero (Sorghum almum) y pasto King Grass cultivar Taiwán (Pennisetum purpureum), una hectárea por cultivo. Previo a la siembra de cada especie, el terreno se surcó a $70 \mathrm{~cm}$ y se adicionó fertilizante al fondo del surco, aplicando el equivalente de $60 \mathrm{~kg}$ de fósforo y $20 \mathrm{~kg}$ de nitrógeno por hectárea.

El maíz se sembró de manera manual a una distancia de $40 \mathrm{~cm}$ entre plantas; se depositaron tres semillas por golpe, para una densidad teórica de 107142 plantas/ha. Dos días posteriores a la siembra, se aplicaron dos kilogramos del herbicida preemergente Atrazina $900 \mathrm{~g} / \mathrm{kg}$ disuelto en 2001 de agua y asperjado con bomba de espalda, para controlar el ciclo vegetativo de las malezas.

En el caso del sorgo negro forrajero, la siembra se efectuó con una cantidad de $20 \mathrm{~kg}$ de semilla/ha (85\% semilla pura germinable).

Para el pasto Taiwán, se procedió a colocar la semilla vegetativa en hileras, colocando los tallos en forma manual con un traslape de $10 \mathrm{~cm}$ en las puntas, para asegurar un brote uniforme a lo largo de los surcos. Luego se procedió a tapar la semilla con la tierra de los entresurcos.

Un mes y medio después de la siembra, se aplicaron $250 \mathrm{~kg} / \mathrm{ha}$ de nitrógeno como nitrato de amonio en todos los cultivos.

El maíz se cosechó a los 116 días, el sorgo negro forrajero a los 114 y el pasto Taiwán a los 112 días. Para cada especie, se cortaron manualmente todas las plantas presentes en seis segmentos de $5 \mathrm{~m}$ lineales, seleccionados aleatoriamente dentro del área cultivada. Tres de los segmentos se cortaron a $15 \mathrm{~cm}$ y los otros tres se cosecharon a $45 \mathrm{~cm}$ sobre el nivel del suelo. Posteriormente, se pesó el material cosechado para estimar la producción de materia verde total; luego las muestras se separaron en tallos y hojas, para pesar cada componente. Seguidamente se cuarteó el material, se tomó una muestra representativa de cada componente y se secó a $60{ }^{\circ} \mathrm{C}$ durante $48 \mathrm{~h}$ hasta alcanzar un peso constante. Finalmente, las muestras se molieron en un molino Wiley (Model \#2, Arthur H. Thomas Co., PA, USA) a 1,0 mm y se determinó el contenido de materia seca (MS) en una estufa a $105{ }^{\circ} \mathrm{C}$, la proteína cruda (PC) por el método de Kjeldahl, el extracto etéreo (EE) y las cenizas totales según los métodos establecidos por la AOAC (2000). La fibra neutro detergente (FDN), la fibra ácido detergente (FAD) y la lignina fueron analizadas por el método de Goering y Van Soest (1970) y Van Soest et al. (1991).

Se empleó un diseño irrestrictamente al azar, con tratamientos en arreglo factorial 3x2, el primer factor: tres variedades de forraje y el segundo: dos alturas de corte. Los datos obtenidos se analizaron con el PROC MIXED del paquete estadístico SAS (SAS, 2009). Las comparaciones se realizaron para las diferentes alturas de cosecha dentro de la misma especie, y para la misma altura para las diferentes especies. Se efectuó la separación de medias en aquellas variables que resultaron significativas $(\mathrm{P}<0,05)$. 


\section{Resultados y discusión}

\section{Rendimiento de forraje verde y seco}

El rendimiento de forraje verde fue diferente $(\mathrm{P}<0,05)$ entre especies a una misma altura de cosecha y dentro de la misma especie cuando se consideraron las diferentes alturas de corte (Cuadro 1). La producción de forraje verde fue siempre mayor cuando la planta se cosechó a una altura de $15 \mathrm{~cm}$ sobre el nivel del suelo.

Cuadro 1. Rendimiento y relación hoja:tallo (H:T) de maíz criollo, sorgo negro forrajero y pasto king grass cultivar Taiwán, cosechados a dos alturas de corte. Cartago, Costa Rica. 2015.

Table 1. Yield and leaf:stem (L:S) ratio in corn, black sorghum, and king grass cultivar Taiwan harvested at two different cutting heights. Cartago, Costa Rica. 2015.

\begin{tabular}{|c|c|c|c|c|c|c|}
\hline & \multicolumn{2}{|c|}{ Maíz criollo } & \multicolumn{2}{|c|}{ Sorgo Negro } & \multicolumn{2}{|c|}{ Taiwán } \\
\hline & $15 \mathrm{~cm}$ & $45 \mathrm{~cm}$ & $15 \mathrm{~cm}$ & $45 \mathrm{~cm}$ & $15 \mathrm{~cm}$ & $45 \mathrm{~cm}$ \\
\hline \multicolumn{7}{|c|}{ Forraje verde, $\mathrm{kg} / \mathrm{ha}$} \\
\hline Hoja & $15524 \mathrm{a}^{2}$ & $11952 b^{2}$ & $8571 \mathrm{a}^{3}$ & $7238 \mathrm{~b}^{3}$ & $21905 \mathrm{a}^{1}$ & $13809 b^{1}$ \\
\hline Tallo & $35761 \mathrm{a}^{2}$ & $28619 b^{1}$ & $22857 a^{3}$ & $15809 b^{2}$ & $49048 \mathrm{a}^{1}$ & $28571 \mathrm{~b}^{1}$ \\
\hline Planta entera & $51286 \mathrm{a}^{2}$ & $40571 b^{2}$ & $31428 a^{3}$ & $23047 b^{3}$ & $70953 a^{1}$ & $42380 b^{1}$ \\
\hline Relación H:T & $0,42^{1}$ & 0,43 & $0,37 b^{2}$ & $0,46 \mathrm{a}$ & $0,45^{1}$ & 0,48 \\
\hline \multicolumn{7}{|c|}{ Forraje seco, $\mathrm{kg} / \mathrm{ha}$} \\
\hline Hoja & $2857 a^{2}$ & $1962 b^{2}$ & $1600 b^{3}$ & $1999 a^{2}$ & $4866 \mathrm{a}^{1}$ & $3230 \mathrm{~b}^{1}$ \\
\hline Tallo & $4381 \mathrm{a}^{3}$ & $3495 b^{2}$ & $5562 \mathrm{a}^{2}$ & $3885 b^{1}$ & $6762 a^{1}$ & $3514 b^{2}$ \\
\hline Planta entera & $7238 a^{2}$ & $5457 \mathrm{~b}^{2}$ & $7162 a^{2}$ & $5885 \mathrm{~b}^{2}$ & $11630 \mathrm{a}^{1}$ & $6742 b^{1}$ \\
\hline Reducción, \% & \multicolumn{2}{|c|}{$24,60^{2}$} & \multicolumn{2}{|c|}{$17,83^{1}$} & \multicolumn{2}{|c|}{$42,03^{3}$} \\
\hline
\end{tabular}

a,b Letra diferente en una misma fila difieren con respecto a la altura de corte dentro de la misma especie $(\mathrm{P}<0,05)$ / Different letters in the same row reflect a difference between different cutting height, within the same specie $(\mathrm{P}<0.05)$.

${ }^{1,2,3}$ Número diferente en una misma fila difieren con respecto a la misma altura al compararse con las otras dos especies $(\mathrm{P}<0,05)$ / Different numbers in the same row, reflect a difference between the same cutting height, when compared to the other two species $(\mathrm{P}<0.05)$.

El cultivar repercutió notablemente sobre los rendimientos productivos; se han reportado producciones promedio de forraje verde de 102,8 t/ha para el maíz criollo y 62,6 t/ha para el maíz híbrido (Elizondo y Boschini, 2002).

El rendimiento de materia seca mostró diferencias significativas $(\mathrm{P}<0,05)$ entre especies a una misma altura de cosecha y dentro de la misma especie cuando se consideró las diferentes alturas de corte.

El pasto Taiwán tuvo ventaja sobre las otras especies estudiadas con rendimientos de $11,6 \mathrm{t} / \mathrm{ha} / \mathrm{corte}$ y $6,7 \mathrm{t} /$ ha/corte, cuando se cosechó a 15 y $45 \mathrm{~cm}$ del suelo, respectivamente. En un estudio realizado con pasto Taiwán se reportaron rendimientos de 80,5 t de forraje verde y 17,5 t MS/ha/corte (Araya y Boschini, 2005), valores superiores a los encontrados en el presente ensayo.

En maíz, materiales híbridos sembrados en la parte alta de Cartago en Costa Rica cosechados a $10 \mathrm{~cm}$ sobre el nivel del suelo, mostraron rendimientos de 88,0 y $11,2 \mathrm{t} / \mathrm{ha}$ de materia verde y seca, respectivamente (Amador y Boschini, 2000). En similares condiciones, el maíz criollo blanco registró rendimientos de 15 a 20 t MS/ha de materia seca (Boschini y Elizondo, 2004b). 
Con referencia al sorgo negro forrajero, Boschini y Elizondo (2005) reportaron producciones de 25,7 y 4,4 t/ ha/corte de materia verde y seca, respectivamente, cosechados a tres centímetros del suelo.

En los Estados Unidos se reportó que al aumentar la altura de corte del forraje, se presentaron disminuciones en el rendimiento de materia seca que variaron entre un 5 y 10\% (Neylon y Kung, 2003). Sin embargo, en el presente estudio las disminuciones estuvieron entre 17 y $42 \%$. Esta disminución es también muy superior a la reportada como el promedio de los once estudios $(7,4 \%)$ resumidos por Wu y Roth (2005). Esta diferencia se debe principalmente a la especie forrajera utilizada, ya que el maíz híbrido es el principal material utilizado en los Estados Unidos para este tipo de estudios. En nuestro país, en una investigación con maíz híbrido, cosechado a 15 o $45 \mathrm{~cm}$ sobre el nivel del suelo, se encontró solamente una disminución del 5\%, cuando se incrementó la altura de cosecha, pasando de 10,9 a 10,4 t MS/ha (Elizondo, 2011).

La relación hoja:tallo (H:T) fue inferior a 1,0 para las tres especies estudiadas; al respecto, Elizondo y Boschini (2002) indicaron que este comportamiento es el esperado cuando las plantas se cosechan a edades superiores a los cien días. Los mismos autores reportaron relaciones H:T superiores a 1,0 cuando la planta se cosechó antes de los setenta días (Elizondo y Boschini, 2001).

El sorgo negro forrajero presentó la relación H:T $(\mathrm{P}<0,05)$ más baja y fue la única especie que presentó un incremento significativo $(\mathrm{P}<0,05)$ en esta variable al aumentar la altura de corte a $45 \mathrm{~cm}$, lo cual es de esperar, ya que al cosechar a una mayor altura se elimina parte del mayor componente presente y, por lo tanto, se mejora la relación. Un incremento como este puede significar mayor calidad nutricional del forraje cosechado, ya que la parte más baja del tallo, que se deja en el campo, es altamente fibroso y lignificado y, por lo tanto, presenta una menor digestibilidad (Tolera y Sundstol, 1999).

En un ensayo con maíz criollo se encontró que la relación H:T fue de $36 \%$ cuando se cosechó a los $15 \mathrm{~cm}$ (Elizondo, 2011). Relaciones H:T de 0,42 y 0,29 para el maíz híbrido y criollo, respectivamente, cosechados a 10 cm del suelo, fueron obtenidas por Elizondo y Boschini (2002).

\section{Composición nutricional}

El valor nutricional de los forrajes, que depende principalmente de la especie, reviste gran importancia en la nutrición del ganado de leche, ya que la ingesta adecuada de nutrientes provenientes del forraje repercute de manera importante sobre la producción de los animales y, por ende, sobre la rentabilidad de la explotación.

\section{Materia seca}

La concentración de materia seca se vio afectada $(\mathrm{P}<0,05)$ principalmente por la especie de forraje. El maíz presentó la menor concentración de materia seca (cercana al 14\%), y solamente el sorgo negro forrajero aumentó la concentración de materia seca $(\mathrm{P}<0,05)$ al incrementar la altura de corte (Cuadro 2). Pese a que el sorgo negro forrajero presentó la mayor concentración de materia seca (superior al 22\%), este fue inferior al 35\% que es el mínimo recomendado para la elaboración de ensilajes (Roth y Heinrichs, 2001).

Se encontraron algunas diferencias significativas $(\mathrm{P}<0,05)$ en la concentración de materia seca en hojas y tallos al comparar la altura de corte entre la misma especie (Cuadro 3); sin embargo, todas las especies presentaron diferencias significativas $(\mathrm{P}<0,05)$ cuando se comparó el contenido de materia seca en los diferentes componentes de la planta entre especies. Se observó que la concentración de materia seca en la hoja fue mayor que la del tallo, sin importar la altura de corte o la especie de forraje. Esto es razonable, ya que de acuerdo con los datos encontrados, la mayor cantidad de agua se encontraba en los tallos de las plantas.

La concentración de materia seca en los forrajes es uno de los aspectos más importantes en la nutrición de rumiantes, ya que es donde se concentran los nutrientes. Sin embargo, la concentración de esta en los forrajes es 
Cuadro 2. Composición nutricional (\%, en base seca) de la planta entera de maíz criollo, sorgo negro forrajero y pasto king grass cultivar Taiwán cosechados a dos alturas de corte. Cartago, Costa Rica. 2015.

Table 2. Nutritional composition (\%, dry matter basis) of the entire plant of corn, black sorghum, and king grass cultivar Taiwan harvested at two different cutting heights. Cartago, Costa Rica. 2015.

\begin{tabular}{lcccccc}
\hline & \multicolumn{2}{c}{ Maíz } & \multicolumn{2}{c}{ Sorgo Negro } & \multicolumn{2}{c}{ Taiwán } \\
\cline { 2 - 7 } & $\mathbf{1 5} \mathbf{~ c m}$ & $\mathbf{4 5} \mathbf{~ c m}$ & $\mathbf{1 5} \mathbf{~ c m}$ & $\mathbf{4 5} \mathbf{~ c m}$ & $\mathbf{1 5} \mathbf{~ c m}$ & $\mathbf{4 5} \mathbf{~ c m}$ \\
\hline Materia seca & $13,94^{3}$ & $13,62^{3}$ & $22,79 \mathrm{~b}^{1}$ & $25,53 \mathrm{a}^{1}$ & $16,20^{2}$ & $16,41^{2}$ \\
Proteína cruda & $9,34^{2}$ & $9,14^{2}$ & $8,24 \mathrm{~b}^{2}$ & $12,90 \mathrm{a}^{1}$ & $9,44^{1}$ & $9,95^{2}$ \\
Cenizas & $8,13^{2}$ & $8,63^{2}$ & $6,19 \mathrm{~b}^{3}$ & $7,94 \mathrm{a}^{2}$ & $13,57^{1}$ & $13,99^{1}$ \\
Extracto etéreo & $3,49^{2}$ & $3,47^{3}$ & $3,22 \mathrm{~b}^{2}$ & $4,10 \mathrm{a}^{2}$ & $5,01^{1}$ & $4,97^{1}$ \\
FND & 61,15 & 61,84 & $63,69 \mathrm{~b}$ & $59,47 \mathrm{a}$ & 65,62 & 64,25 \\
FDA & 39,77 & 41,54 & $43,15 \mathrm{~b}$ & $37,30 \mathrm{a}$ & 45,75 & 44,67 \\
Lignina & $6,85^{2}$ & $6,93^{2}$ & $7,73 \mathrm{~b}^{2}$ & $6,36 \mathrm{a}^{2}$ & $4,96^{1}$ & $4,70^{1}$ \\
\hline
\end{tabular}

FND: fibra neutro detergente (Neutral detergent fiber). FAD: fibra ácido detergente (Acid detergent fiber). $a, b$ Letra diferente en una misma fila difieren con respecto a la misma especie cosechada a diferente altura $(\mathrm{P}<0,05) /$ Different letters in the same row differ between different cutting heights, within the same specie $(\mathrm{P}<0.05)$.

$1,2,3$ Número diferente en una misma fila, difieren con respecto a la misma altura al compararse entre especies $(\mathrm{P}<0,05$ / Different numbers in the same row differ between the same cutting height, when compared to other species $(\mathrm{P}<0.05)$.

Cuadro 3. Composición nutricional (\%, en base seca) en hojas y tallos de maíz criollo, sorgo negro forrajero y pasto king grass cultivar Taiwán cosechados a diferentes alturas de corte. Cartago, Costa Rica. 2015.

Table 3. Nutritional composition (\%, dry matter basis) of leaves and stems of corn, black sorghum, and king grass cultivar Taiwan harvested at two different cutting heights. Cartago, Costa Rica. 2015.

\begin{tabular}{|c|c|c|c|c|c|c|c|c|c|c|c|c|}
\hline & \multicolumn{4}{|c|}{ Maíz } & \multicolumn{4}{|c|}{ Sorgo Negro } & \multicolumn{4}{|c|}{ Taiwán } \\
\hline & \multicolumn{2}{|c|}{ Hoja } & \multicolumn{2}{|c|}{ Tallo } & \multicolumn{2}{|c|}{ Hoja } & \multicolumn{2}{|c|}{ Tallo } & \multicolumn{2}{|c|}{ Hoja } & \multicolumn{2}{|c|}{ Tallo } \\
\hline & $15 \mathrm{~cm}$ & $45 \mathrm{~cm}$ & $15 \mathrm{~cm}$ & $45 \mathrm{~cm}$ & $15 \mathrm{~cm}$ & $45 \mathrm{~cm}$ & $15 \mathrm{~cm}$ & $45 \mathrm{~cm}$ & $15 \mathrm{~cm}$ & $45 \mathrm{~cm}$ & $15 \mathrm{~cm}$ & $45 \mathrm{~cm}$ \\
\hline Materia seca & $18,33 a^{3}$ & $16,74 b^{3}$ & $11,90^{2}$ & $12,36^{2}$ & $27,23^{1}$ & $27,71^{1}$ & $24,32^{1}$ & $24,67^{1}$ & $21,96 b^{2}$ & $23,44 a^{2}$ & $13,68^{2}$ & $12,73^{2}$ \\
\hline Proteína cruda & $13,29^{2}$ & $13,04^{2}$ & 6,91 & 7,00 & $15,51 b^{1}$ & $16,55 \mathrm{a}^{1}$ & 6,23 & 6,37 & $13,45^{2}$ & $13,50^{2}$ & 6,59 & 6,57 \\
\hline Cenizas & $9,76^{2}$ & $10,17^{2}$ & $7,10^{2}$ & $7,78^{2}$ & $8,62^{3}$ & $8,86^{3}$ & $5,57^{3}$ & $6,24^{3}$ & $16,51^{1}$ & $16,62^{1}$ & $11,47^{1}$ & $11,54^{1}$ \\
\hline Extracto etéreo & 3,88 & $3,39^{2}$ & $3,17^{2}$ & $3,47^{2}$ & 4,91 & $4,71^{1}$ & $3,16^{2}$ & $3,18^{2}$ & $4,56 \mathrm{~b}$ & $5,45 \mathrm{a}^{1}$ & $5,26 a^{1}$ & $4,40 b^{1}$ \\
\hline FND & $65,65 \mathrm{~b}^{2}$ & $59,69 a$ & $58,36^{1}$ & $62,93^{1}$ & $55,71^{1}$ & 56,76 & $65,79^{2}$ & $64,98^{1}$ & $58,44^{1}$ & 59,10 & $70,77^{3}$ & $69,102^{2}$ \\
\hline FDA & $41,57 b^{2}$ & $37,96 \mathrm{a}^{2}$ & $38,62 \mathrm{a}^{1}$ & $43,48 b^{1}$ & $35,48^{1}$ & $34,42^{1}$ & $45,38 b^{2}$ & $42,75 a^{1}$ & $41,13^{2}$ & $41,07^{3}$ & $49,07^{3}$ & $48,02^{2}$ \\
\hline Lignina & $7,29 b^{3}$ & $6,34 a^{2}$ & $6,53^{1}$ & $7,26^{2}$ & $5,89^{2}$ & $6,36^{2}$ & $8,19 b^{2}$ & $6,25 \mathrm{a}^{1}$ & $3,35^{1}$ & $3,38^{1}$ & $6,13^{1}$ & $5,97^{1}$ \\
\hline
\end{tabular}

FND: fibra neutro detergente (Neutral detergent fiber). FAD: fibra ácido detergente (Acid detergent fiber).

$a, b$ Letra diferente en una misma fila difieren con respecto al componente (hoja o tallo) de la misma especie cosechada a diferente altura $(\mathrm{P}<0,05)$ / Different letters in the same row reflect a difference according to a different cutting height, within the plant component $($ leaf or stem) in the same specie $(\mathrm{P}<0.05)$.

${ }^{1,2,3}$ Número diferente en una misma fila, difieren con respecto a la misma altura al compararse entre especies dentro del mismo componente $(\mathrm{P}<0,05) /$ Different numbers in the same row reflect a difference when compared between the same cutting height and other species within the same plant component $(\mathrm{P}<0.05)$. 
altamente variable y depende de una serie de factores primarios como la especie, la parte de la planta, la edad de corte o rebrote, y de una serie de factores secundarios como el periodo del año, la fertilidad del suelo, el manejo y las condiciones climáticas a las cuales se hallan expuestos (Bernal, 1991). Así por ejemplo, Lloveras e Iglesias (2001), demostraron que la concentración de materia seca aumenta al incrementarse la edad de cosecha. Estas diferencias revisten gran importancia al considerar que la especie de forraje a utilizar puede causar una diferencia importante en cuanto a la contribución de nutrientes que haga al sistema de producción.

Las concentraciones de materia seca determinadas en este experimento fueron muy similares a las reportadas por Araya y Boschini (2005), quienes obtuvieron valores de 23,79 y 17,28\% para hojas y tallos de pasto Taiwán, respectivamente. Concentraciones de materia seca muy parecidos a los encontrados en este ensayo para maíz criollo en la zona de Cartago fueron obtenidos por Elizondo (2011). Los valores de materia seca obtenidos también fueron muy similares a los obtenidos en otras investigaciones en el país (Elizondo y Boschini, 2001; Elizondo y Boschini, 2003; Boschini y Elizondo, 2004a).

En un estudio realizado con sorgo negro forrajero, cosechado a los 119 días, se reportaron concentraciones de materia seca de 21,09; 14,49 y 17,01\% para hojas, tallo y planta entera, respectivamente (Boschini y Elizondo, 2005).

\section{Proteína cruda}

La concentración de proteína cruda varió, entre especie forrajera, mientras que la altura de cosecha tuvo poco efecto sobre su concentración. El sorgo negro forrajero fue el único que mostró un incremento significativo $(\mathrm{P}<0,05)$ en el contenido de proteína cruda de la planta entera cuando se aumentó la altura de cosecha, pasando de 8,24 a 12,90\% (Cuadro 2). Este incremento se debe a que al mejorar la relación H:T, aumentó la concentración de proteína cruda, ya que este nutriente se encuentra en mayor concentración en las hojas de la planta. Es importante indicar que la calidad nutricional de los tallos de los forrajes a una edad temprana es generalmente alta, incluso puede llegar a ser mayor que la de las hojas (Minson, 1990); sin embargo, su calidad disminuye a una tasa mayor que la de las hojas, especialmente conforme las plantas alcanzan la madurez (Nelson y Moser, 1994).

El sorgo negro forrajero presentó la mayor concentración de proteína cruda cuando se cosechó a $45 \mathrm{~cm}$, mientras que el maíz y el pasto Taiwán presentaron concentraciones superiores al $9 \%$ a la misma altura de corte. Tal como se discutió anteriormente, el perfil nutricional es muy variable y la especie es el factor que tiene mayor peso sobre dicha variabilidad.

Al considerar las diferentes fracciones de la planta (Cuadro 3), la concentración de proteína cruda mostró poca variación en hojas y tallos al considerar las diferentes alturas de corte dentro de la misma especie. El sorgo negro forrajero presentó la mayor concentración de proteína cruda en las hojas cuando se cosechó a $45 \mathrm{~cm}$ sobre el nivel del suelo. El contenido de este nutrimento fue siempre superior en hoja que en tallo. Esta situación reviste gran importancia, especialmente al considerar las implicaciones que tiene el aumentar la altura de corte, ya que esto implica material de mayor calidad para los animales. La concentración de proteína cruda en los tallos de las tres especies fue menor al 7\%, mínimo recomendado para mantener un adecuado aporte para los microorganismos ruminales (Sánchez et al., 1998).

En un ensayo llevado a cabo en los Estados Unidos, donde se estudió la composición nutricional de tres híbridos de maíz cosechados a 12,7 o 45,7 cm sobre el nivel del suelo, se encontró que incrementar la altura de corte aumentó significativamente la concentración de materia seca, pero disminuyó la concentración de proteína cruda (Neylon y Kung, 2003). Por su parte, Kung et al. (2008) no obtuvieron diferencias significativas ( $>>0,05)$ entre tratamientos al utilizar alturas de corte de 10 y $50 \mathrm{~cm}$, pero señalaron incrementos numéricos en la concentración de MS y PC.

Los valores de proteína cruda encontrados para las hojas y tallos de maíz fueron inferiores a los hallados por Elizondo (2011), quien reportó concentraciones superiores a 17 y $7 \%$ para hojas y tallos, respectivamente. Con 
respecto al pasto Taiwán, los valores obtenidos fueron muy similares a los reportados por Araya y Boschini (2005), con concentraciones de 13,69, 7,29 y 9,73\% para las hojas, tallo y planta entera, respectivamente. Con respecto al sorgo negro forrajero, en un estudio se informaron concentraciones de 14,64, 5,95 y 10,02\% para hojas, tallo y planta entera, respectivamente, valores inferiores a los obtenidos en este estudio (Boschini y Elizondo, 2005).

\section{Cenizas}

La concentración de cenizas aumentó significativamente $(\mathrm{P}<0,05)$ en la planta entera al aumentar la altura de corte en el sorgo negro forrajero. El pasto Taiwán fue el que presentó la mayor concentración $(\mathrm{P}<0,05)$, de las tres especies estudiadas, mientras que el sorgo negro forrajero mostró el menor valor para este nutriente (Cuadro 2).

No se encontraron diferencias significativas $(\mathrm{P}>0,05)$ dentro de una misma especie al variar la altura de cosecha con respecto a las diferentes fracciones de la planta (Cuadro 3); sin embargo, si se encontraron diferencias significativas $(\mathrm{P}<0,05)$ entre especies.

Los valores de cenizas encontrados en las hojas de maíz fueron muy similares a las reportadas por Elizondo (2011) y menores a los reportados por Araya y Boschini (2005) para el pasto Taiwán, quienes obtuvieron concentraciones superiores al $20 \%$ en hojas y cercanas a $13 \%$ en tallos. Boschini y Elizondo (2005) reportaron concentraciones de cenizas de 10,57 y $9,40 \%$ en sorgo negro forrajero para hojas y tallos, respectivamente.

\section{Extracto etéreo}

Al considerar la planta entera, solo para el sorgo negro forrajero el extracto etéreo aumentó significativamente $(\mathrm{P}<0,05)$ al incrementar la altura de cosecha. Las tres especies de forraje estudiadas presentaron concentraciones significativamente diferentes $(\mathrm{P}<0,05)$ entre sí; fue el pasto Taiwán el que presentó la mayor concentración, con un valor cercano al $5 \%$ (Cuadro 2).

En las diferentes fracciones de la planta, solamente con el pasto Taiwán se encontraron diferencias significativas $(\mathrm{P}<0,05)$ al variar la altura de cosecha, y también se encontraron diferencias significativas entre especies. Los valores obtenidos para el extracto etéreo variaron entre 3,16 y 5,45\% (Cuadro 3). Estos valores fueron superiores a los reportados por Villalobos y Arce (2014) y Salazar (2007) en pasto estrella africana (Cynodon nlemfuensis) de 2,67 y $0,91 \%$, respectivamente.

Los forrajes proveen una cantidad importante de grasas y ácidos grasos a la dieta de los rumiantes (Harfoot y Hazlewood, 1988). De acuerdo con Bernal (1991), el contenido de grasa en las hojas puede variar entre 3 y $10 \%$ con base en la concentración de materia seca, y declina con la edad del forraje. Generalmente, se localizan en los cloroplastos de las hojas (Boufaïed et al., 1995), y se encuentran principalmente como glicolípidos y fosfolípidos (Harfoot y Hazlewood, 1988). Su concentración en los forrajes depende de múltiples factores como la especie (Harfoot y Hazlewood, 1988; Elgersma et al., 2005), la edad de corte o rebrote (Bauchart et al., 1984; Elgersma et al., 2005), la época del año, efectos climáticos como cantidad de horas luz, intensidad lumínica, temperatura, humedad, y características de la pastura (Elgersma et al., 2005), entre otros. Además, se indica que la concentración de este nutriente se incrementa por efecto de la fertilización nitrogenada (Boufaïed et al., 1995).

El presente estudio no buscó alterar el perfil graso de los forrajes, pero es importante agregar que alterar la concentración de grasa en este componente de la dieta, puede ser la manera más económica de cambiar las características de ácidos grasos de la carne o leche producida por los rumiantes, ya que manipular la composición de lípidos en los forrajes puede mejorar el valor nutricional de los forrajes y las características nutricionales de los productos animales consumidos por los humanos. 


\section{Componentes de la pared celular}

El contenido de pared celular fue similar entre las especies evaluadas, sin embargo, el sorgo negro forrajero tuvo contenidos menores $(\mathrm{P}<0,05)$ de fibra neutro y fibra ácida detergente cuando se aumentó la altura de corte mayor (Cuadro 2). Situación similar fue reportada en maíz híbrido para ensilaje (Neylon y Kung, 2003). Esta situación es debida a que se deja en el campo la parte del tallo que presenta mayor cantidad de componentes de la pared celular.

Encontrar diferencias significativas entre cultivares con respecto a la calidad nutricional, fue consistente con trabajos llevados a cabo en Costa Rica; así por ejemplo, la concentración de FND para la planta entera de maíz fue superior al 70\%, y fue mayor en el forraje de maíz criollo $(75,46 \%)$ que en el híbrido $(71,78 \%$ ) (Elizondo y Boschini, 2003).

En un ensayo llevado a cabo en sorgo negro forrajero, se reportaron concentraciones de FND y FAD en la planta entera de 77,16 y 45,22\%, respectivamente (Boschini y Elizondo, 2005).

En el ensayo sobre la composición nutricional de tres híbridos de maíz cosechados a 12,7 o 45,7 cm sobre el nivel del suelo, se encontró que incrementar la altura de corte disminuyó la concentración de FAD, y no afectó la concentración de FDN (P>0,05) (Neylon y Kung, 2003); en este estudio también hubo un incremento moderado en la concentración de almidones y en la digestibilidad in vitro de la FDN. Por su parte, Bernard et al. (2004) compararon alturas de cosecha de 10 y $30 \mathrm{~cm}$ sobre el nivel del suelo y encontraron que a una altura mayor disminuyó la concentración de FAD $(\mathrm{P}<0,01)$, pero no obtuvieron diferencias en la concentración de FDN o en la digestibilidad verdadera in vitro de la materia seca. En otro estudio no se encontraron diferencias significativas $(\mathrm{P}>0,05)$ entre tratamientos al utilizar alturas de corte de 10 y $50 \mathrm{~cm}$, pero se señalaron disminuciones en la concentración de la FDN y FAD (Kung et al., 2008).

Con respecto a las diferentes fracciones de la planta, se encontraron diferencias significativas para la FND y la FAD entre las diferentes especies (Cuadro 3). La hoja del forraje de maíz presentó la mayor concentración de FND $(\mathrm{P}<0,05)$ cuando se cosechó a $15 \mathrm{~cm}$, mientras que la fracción de hojas del sorgo negro forrajero presentó la menor concentración de FDA cuando se cosechó a 15 o $45 \mathrm{~cm}$.

La observación más común es que conforme madura el forraje, la relación H:T disminuye (más tallo, menos hojas), y como resultado la digestibilidad de la FND se reduce, debido a que una mayor proporción de la FDN está asociada al tejido del tallo (Hofmann et al., 2003).

Al comparar la concentración de componentes de la pared celular en diferentes especies forrajeras, se determinó que dicha concentración era menor en hojas que en tallos, y la calidad nutricional de la parte inferior de los tallos disminuyó significativamente con la madurez (Buxton y Hornstein, 1986).

Las diferencias encontradas para la concentración de los componentes de la pared celular con respecto a las diferentes especies, debe ser tomado en cuenta para poder utilizar aquellas que mejor complementen las necesidades nutricionales de los animales, ya que contenidos altos de componentes de la pared celular repercute negativamente sobre el consumo y la digestibilidad de la materia seca, lo que a su vez origina bajos rendimientos productivos en los animales (NRC, 2001).

\section{Lignina}

Cuando se consideró la planta entera, la concentración de lignina disminuyó significativamente $(\mathrm{P}<0,05)$ al aumentar la altura de corte en el sorgo negro forrajero. El pasto Taiwán fue el que presentó la menor concentración con valores cercanos al 5\% (Cuadro 2).

Al considerar las diferentes fracciones de la planta, la concentración de lignina presentó diferencias significativas $(\mathrm{P}<0,05)$ entre especies y dentro de la misma especie cuando se varió la altura de corte. El pasto 
Taiwán presentó la menor concentración de este componente, tanto en hojas como en tallos. Se han reportado concentraciones de lignina de 4,43 y $5,38 \%$ en sorgo negro forrajero para hoja y tallo, respectivamente (Boschini y Elizondo, 2005).

La lignina le confiere rigidez a la planta y, por lo tanto, aumenta conforme avanza la madurez de la misma; su contenido, composición y localización están determinados genéticamente, pero puede verse influenciados por factores ambientales como la temperatura (Boon et al., 2005).

El proceso de lignificación reduce considerablemente la degradación de carbohidratos fibrosos a nivel ruminal y en consecuencia, hay una menor disponibilidad energética para los animales (NRC, 2001).

A pesar de que en el presente ensayo no se obtuvo una disminución generalizada en la concentración de lignina al aumentar la altura de cosecha del forraje, la calidad superior de forraje de maíz cosechado a alturas mayores ha sido documentada en diferentes estudios (Oba y Allen, 1999; Ebling y Kung, 2004), donde se ha indicado que la menor concentración de lignina es el principal factor responsable de los aumentos en la digestibilidad de la fibra.

Los resultados del presente ensayo parecen indicar que la razón por la que solamente el sorgo negro forrajero presentó diferencias significativas en sus diferentes componentes nutricionales al aumentar la altura de cosecha de la planta, fue el aumento significativo $(\mathrm{P}<0,05)$ que presentó la relación H:T. El tejido de las hojas de las plantas es generalmente de mayor calidad que el de los tallos. La calidad nutricional de las hojas y los tallos de la mayoría de plantas forrajeras son relativamente similares a edades jóvenes, pero conforme el tejido envejece, la calidad nutricional de las hojas disminuye a una tasa menor; esto se debe principalmente a que las células epidérmicas y de fibra en el tallo forman paredes celulares secundarias gruesas y el tejido se vuelve altamente lignificado conforme envejece (Nelson y Moser, 1994).

Algunos autores, en cuyos ensayos no se han encontrado diferencias significativas, han sugerido la posibilidad de que la porción baja de las plantas de algunas variedades o especies forrajeras pueden ser de similar composición nutricional que la porción alta, y por esta razón, el aumentar la altura de cosecha no ejerce un efecto sobre la composición nutricional (Bernard et al., 2004). Otro aspecto de importancia a considerar es que la no respuesta sobre las variables estudiadas pudo estar influenciada por muchos otros factores, además de la altura de corte. Por ejemplo, es bien conocido que las condiciones climáticas y la cantidad de humedad afecta considerablemente el contenido de materia seca y la composición nutricional de los forrajes (Bernard et al., 2004). De la misma manera, la fertilidad del suelo y la aplicación de minerales afectan la producción de biomasa y su valor bromatológico.

En otro estudio, Lounglawan et al. (2014) concluyeron que la altura de corte $(5,10 \mathrm{o} 15 \mathrm{~cm})$ sobre el nivel del suelo no tuvo ningún efecto sobre el rendimiento de materia seca o sobre la calidad nutricional del pasto King grass (Pennisetum purpureum x Pennisetum americanum), sin embargo, los incrementos de altura de corte en dicho ensayo fueron de solamente 5 o $10 \mathrm{~cm}$.

\section{Literatura citada}

Amador, A.L., y C. Boschini. 2000. Fenología productiva y nutricional de maíz para la producción de forraje. Agron. Mesoam. 11(1):171-177.

AOAC (Association of Official Analytical Chemistry). 2000. Official methods of analysis. 17 ${ }^{\text {th }}$ ed. AOAC, WA, USA.

Araya, M., y C. Boschini. 2005. Producción de forraje y calidad nutricional de variedades de Pennisetum purpureum en la meseta central de Costa Rica. Agron. Mesoam. 16:37-43.

Bauchart, D., R. Vérité, and B. Remond. 1984. Long-chain fatty acid digestion in lactating cows fed fresh grass from spring to autumn. Can. J. Anim. Sci. 64:330-331.

Bernal, J. 1991. Pastos y forrajes tropicales: producción y manejo. $2^{\text {da }}$ ed. Banco Ganadero, COL. 
Bernard, J.K., J.W. West, D.S. Trammell, and G.H. Cross. 2004. Influence of corn variety and cutting height on nutritive value of silage fed to lactating dairy cows. J. Dairy Sci. 87:2172-2176.

Boon, E.J., F.M. Engels, P.C. Struik, and J.W. Cone. 2005. Stem characteristics of two forage maize (Zea mays L.) cultivars varying in whole plant digestibility. II. Relation between in vitro rumen fermentation characteristics and anatomical and chemical features within a single internode. NJAS 53:87-109.

Boschini, C., y J. Elizondo. 2004a. Desarrollo productivo y cualitativo de maíz híbrido para ensilaje. Agron. Mesoam. 15:31-37.

Boschini, C., y J. Elizondo. 2004b. Rendimiento de forraje de dos materiales genéticos de maíz (Zea mays L.) sembrados a diferentes distancias de siembra. Agron. Trop. 34:87-92.

Boschini, C., y J. Elizondo. 2005. Determinación de la calidad y la producción de sorgo negro forrajero (Sorghum almum) en edades para ensilar. Agron. Mesoam. 16:29-36.

Boufaïed, H., P. Chouinard, G. Tremblay, H. Petit, R. Michaud, and G. Bélanger. 1995. Fatty acids in forages. I. Factors affecting concentrations. Can. J. Anim. Sci. 83:501-511.

Buxton, D., and J. Hornstein. 1986. Cell wall concentration and components of stratified canopies of alfalfa, birdsfoot trefoil, and red clover. Crop Sci. 26:180-184.

Buxton, D., and S. Fales. 1994. Plant environment and quality. In: G. Fahey, editor, Forage quality, evaluation, and utilization. American Society of Agronomy, Madison, WI, USA. p. 155-199.

Ebling, T.L., and L. Kung. 2004. A comparison of processed conventional corn silage to unprocessed and processed brown midrib corn silage on intake, digestion, and milk production by dairy cows. J. Dairy Sci. 87:2519.

Elgersma, A., P. Maudet, I. Witkowska, and A. Wever. 2005. Effects of nitrogen fertilisation and regrowth period on fatty acid concentrations in perennial ryegrass (Lolium perenne L.). Ann. Appl. Biol. 147:145-152.

Elizondo, J. 2011. Influencia de la variedad y altura de cosecha sobre el rendimiento y valor nutritivo de maíz para ensilaje. Agron. Costarricense 35(2):105-111.

Elizondo, J., y C. Boschini. 2001. Efecto de la densidad de siembra sobre el rendimiento y calidad del forraje de maíz. Agron. Mesoam. 12:181-187.

Elizondo, J., y C. Boschini. 2002. Producción de forraje con maíz criollo y maíz híbrido. Agron. Mesoam. 13:13-17.

Elizondo, J., y C. Boschini. 2003. Valoración nutricional de dos variedades de maíz usadas en la producción de forraje para bovinos. Pastos y Forrajes 26:347-353.

Goering, H., and P. Van Soest. 1970. Forage fiber analysis (Apparatus, reagents, procedures and some applications). Agricultural Handbook No. 379. ARS-USDA, WA, USA.

Harfoot, C.G., and G.P. Hazlewood. 1988. Lipid metabolism in the rumen. In: P.N. Hobson, editor, The rumen microbial ecosystem. Elsevier Applied Science, NY, USA. p. 285-322.

Hoffman, P.C., K.L. Lundberg, L.M. Bauman, and R. Shaver. 2003. In vitro NDF digestibility of forages: The 30 vs. 48 hour debate. Focus on Forage 5(16):1-3.

Kung, L., B.M. Moulder, C.M. Mulrooney, R.S. Teller, and R.J. Schmidt. 2008. The effect of silage cutting height on the nutritive value of a normal corn silage hybrid compared with brown midrib corn silage fed to lactating cows. J. Dairy Sci. 91:1451-1457.

Lounglawan, P., W. Lounglawan, and W. Suksombat. 2014. Effect of cutting interval and cutting height on yield and chemical composition of king napier grass (Pennisetum purpureum x Pennisetum americanum). APCBEE Procedia 8:27-31. 
Lloveras, J., and I. Iglesias. 2001. Morphological development and forage quality changes in crimson clover (Trifolium incarnatum L.). Grass Forage Sci. 56:395-404.

Minson, D. 1990. Forage in ruminant nutrition. Academic Press Inc. Sand Diego, CA. USA.

NRC (National Research Council). 2001. Nutrient requirements of dairy cattle. $7^{\text {th }}$ rev. ed. National Academy Press, WA, USA.

Nelson, C., and L. Moser. 1994. Plant factors affecting forage quality. In: G. Fahey, editor, Forage quality, evaluation, and utilization. American Society of Agronomy, Madison, WI, USA.

Neylon, J.M., and L. Kung. 2003. Effects of cutting height and maturity on the nutritive value of corn silage for lactating cows. J. Dairy Sci. 86:2163-2169.

Oba, A., and M.S. Allen. 1999. Evaluation of the importance of the digestibility of neutral detergent fiber from forage: effects on dry matter intake and milk yield of dairy cows. J. Dairy Sci. 82:589-596.

Roth, G.W., and A.J. Heinrichs. 2001. Corn silage production and managements. Agronomy Facts 18. The Pennsylvania State University, PA, USA.

Salazar, S. 2007. Disponibilidad de biomasa y valor nutricional del pasto estrella africana (Cynodon nlemfuensis) en el distrito de Quesada, cantón de San Carlos. Tesis Lic, Universidad de Costa Rica, San José, CRC.

Sánchez, J., L. Piedra, y H. Soto. 1998. Calidad nutricional de los forrajes en zonas con niveles bajos de producción de leche, en la zona norte de Costa Rica. Agron. Costarricense 22(1):69-76.

SAS. 2009. SAS/STAT Software. Release 9.2. SAS Institute Inc., Cary, NC, USA.

Sheaffer, C., P. Seguin, and G. Cuomo. 1998. Sward characteristics and management effects on cool-season grass forage quality. In: J. Cherney, and D. Cherney, editors, Grass for dairy cattle. $2^{\text {nd }}$ ed. CABI Publishing, Oxon, GBR. p. 73-100.

Tolera, A., and F. Sundstol. 1999. Morphological fractions of maize stover harvested at different stages of grain maturity and nutritive value of different fractions of stover. Anim. Feed Sci. Tech. 81:1-16.

Van Soest, P., J. Robertson, and B. Lewis. 1991. Methods for dietary fiber, neutral detergent fiber and non starch polysaccharides in relation to animal nutrition. J. Dairy Sci. 74:3583-3597.

Vásquez, A. 1982. Estudio detallado de los suelos de la Estación Experimental de Ganado Lechero El Alto. Tesis Lic., Universidad de Costa Rica, San José, CRC.

Villalobos, L., y J. Arce. 2014. Evaluación agronómica y nutricional del pasto estrella africana (Cynodon nlemfuensis) en la zona de Monteverde, Puntarenas, Costa Rica. II. Valor nutricional. Agron. Costarricense 38(1):133-145.

Villareal, M., R.C. Cochran, A. Rojas, O. Murillo, H. Muñoz, and M. Poore. 2006. Effect of supplementation with pelleted citrus pulp on digestibility and intake in beef cattle fed a tropical grass-based diet (Cynodon nlemfuensis). Anim. Feed Sci. Technol. 125:163-173.

Wu, Z., and G. Roth. 2005. Considerations in managing cutting height of corn silage. The Pennsylvania State University, PA, USA. 